\title{
Binary Systems As Gravitational Wave Sources
}

\author{
O. Köse ${ }^{1}$ and K. Yakut ${ }^{1,2}$ \\ ${ }^{1}$ Department of Astronomy and Space Sciences, University of Ege, 35100, İzmir, Turkey \\ ${ }^{2}$ Institute of Astronomy, University of Cambridge, Madingley Road, Cambridge CB3 0HA, UK
}

\begin{abstract}
Binary systems with compact components and some newly discovered systems with relatively short orbital periods are studied as gravitational-wave sources. The gravitational wave amplitudes of these systems have been compared with the limit of gravitational wave interferometers (e.g. LISA, LIGO, and VIRGO).
\end{abstract}

Keywords. gravitation, gravitational waves, stars: binaries : close

\section{Introduction}

Gravitational waves (GWs), unlike electromagnetic waves, are propagating ripples in space-time, according to Einstein's General Relativity (GR) (Einstein 1916). GR predicts the existence of GWs that travel at the speed of light with two polarization states, $\mathrm{h}(+)$ and $\mathrm{h}(\mathrm{x})$. Relativistic binary systems (e.g. neutron binary systems) can propagate GW in the observable frequency range (Phinney 1991, Nelemans et al. 2001 ). GWs, therefore, can be detected with ground-based GW detectors such as LIGO, VIRGO, GEO 600, TAMA 300, and future planned space-based laser interferometer, LISA.

\section{Gravitational wave amplitudes and frequencies of binaries}

Starting from the linearized Einstein field equation, one can obtain the gravitational field equation. Following straightforward calculations, we obtain the GW amplitude (h)

$$
h \simeq 2.5 \times 10^{-22} M_{1} M_{2} M^{-1 / 3} D^{-1} f^{2 / 3}
$$

where $\mathrm{f}$ is the frequency, $\mathrm{D}$ is the distance of source in $\mathrm{Mpc}$, and $\mathrm{M}$ is the total mass in solar units.

Close binary systems with compact components lose energy by GWs (Paczynski \& Sienkiewicz 1981, Verbunt \& Zwaan 1981, Yakut et al. 2008, Kalomeni 2010). Studying GWs, it is possible to detect binary systems and double black hole binaries, if they exist. A few detectors have been improved to detect binaries consisting of ultra compact objects. The amplitude of a binary can be measured with Eq. (1). Gravitational frequencies and amplitudes of some binaries are listed in Table 1. In Fig. 1, we show frequencies and amplitudes of these binary systems and detectors. Apart from AM CVn systems and binaries with neutron star components, some hypothetical binary systems are also added. These systems are supposed to be $\mathrm{NS}+\mathrm{NS}, \mathrm{NS}+\mathrm{BS}$, and $\mathrm{BH}+\mathrm{BH}$ binaries. It is apparent from the figure that these objects lies within the observation limit of LISA satellite. Massive black hole binary systems are also added.

Fig. 1 shows that high mass binary systems with small separation (short period) emit more waves that make them easy to detect. The distance of the target is also crucial for the detection limit of detectors. We still continue to analyze close binaries that are 


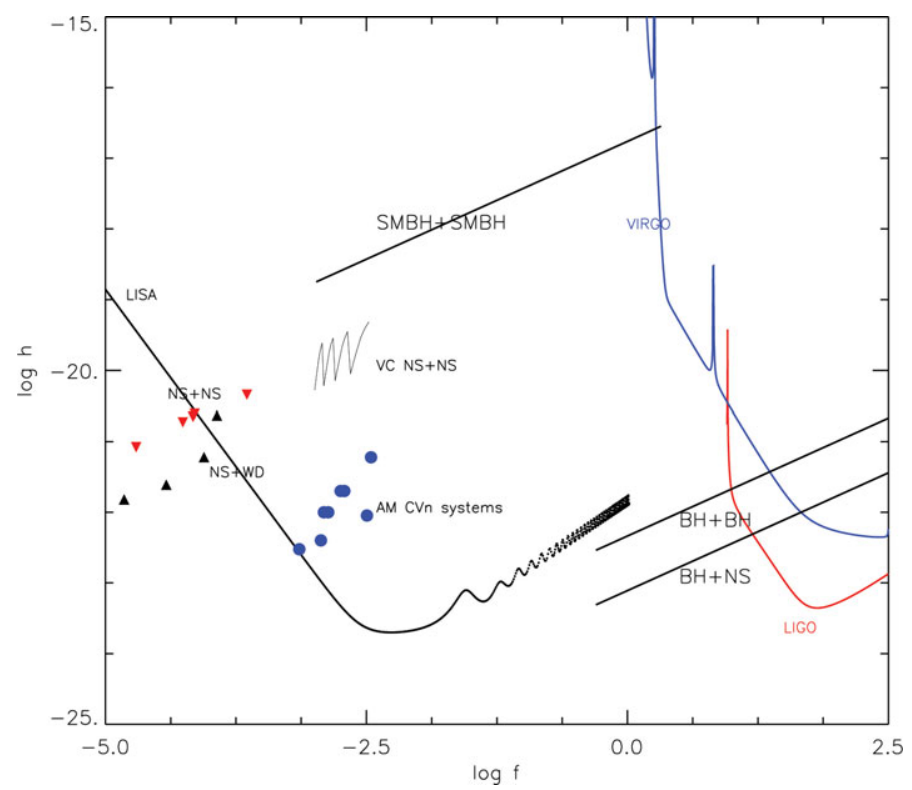

Figure 1. Evolution of some binaries and sensitivities of ground based (LIGO, VIRGO) and space based (LISA) detectors in frequency (f) vs. GW amplitude (h) diagram.

Table 1. GW amplitude and frequency of selected binary systems.

\begin{tabular}{llllll}
\hline & Type & $\mathrm{P}(\min )$ & $\mathrm{M}_{1}, \mathrm{M}_{2}$ & $\log (f)$ & $\log (h)$ \\
\hline V523 Cas & MS+MS & 337 & $0.75,0.38$ & 1.2 & -20 \\
J18073024+4551325 & MS+WD & 279 & $0.60,0.40$ & -3.9 & -21 \\
AM CVn & WD+WD & 17.1 & $0.68,0.12$ & -3.0 & -20.5 \\
XTE J2123-058 & NS+MS & 357 & $1.46,0.53$ & -4.0 & -20.9 \\
PSR J0737-3039 & NS+NS & 147 & $1.25,1.34$ & -3.6 & -20.3 \\
SS433 & BH+MS & 18835 & 16,22 & -5.8 & -19.8 \\
\hline
\end{tabular}

observed with the Kepler satellite in order to catalogue possible sources to study with this technique (Köse and Yakut 2012).

\section{Acknowledgements}

This study was supported by the Turkish Scientific and Research Council (TÜBİTAK 109T047 and 111T270) and Ege University Research Fund. KY acknowledges support by the Turkish Academy of Sciences (TÜBA).

\section{References}

Einstein, A. 1916, Annalender Physik, 354, 769

Kalomeni, B.: 2010, IAU Symposium, 262, 362.

Köse, O. \& Yakut, K.: 2012, in preparation.

Nelemans, G., Yungelson, L. R., \& Portegies Zwart, S. F.: 2001, A\&A, 375, 890.

Paczynski, B. \& Sienkiewicz, R.: 1981, ApJ, 248, L27.

Phinney, E. S.: 1991, ApJ, 380, L17.

Verbunt, F. \& Zwaan, C.: 1981, A\&A, 100, L7.

Yakut, K., Kalomeni, B., \& Tout, C. A.: 2008, ArXiv e-prints, arXiv:0811.0455. 